\title{
State-selective electric-field ionization of Rydberg positronium
}

\author{
A. M. Alonso, L. Gurung, B. A. D. Sukra, S. D. Hogan, and D. B. Cassidy \\ Department of Physics and Astronomy, University College London, Gower Street, London WC1E 6BT, United Kingdom
}

(Received 15 September 2018; published 15 November 2018)

\begin{abstract}
We report experiments in which positronium (Ps) atoms, optically excited to Rydberg states with principal quantum numbers $n$ in the range 18-25, were selectively ionized by both static and pulsed electric fields. The experiments were modeled using Monte Carlo simulations that include tunnel ionization rates calculated for hydrogen and scaled by the Ps reduced mass. Our measurements exhibit a small disagreement with the calculated tunnel ionization rates. Despite this we show that the electric fields in which different Ps states are ionized are sufficiently separated to allow selective field-ionization methods to be used in typical experimental conditions.
\end{abstract}

DOI: 10.1103/PhysRevA.98.053417

\section{INTRODUCTION}

Almost ${ }^{1}$ all atoms and molecules possess an infinite series of excited electronic states, known as Rydberg states [3]. Atoms and molecules excited to Rydberg states can be manipulated using inhomogeneous electric fields [4], and are of interest in a variety of experimental areas (see, e.g., Refs. [5-9]).

The utility of Rydberg systems generally derives from the fact that they have exaggerated, state-dependent properties such as size, polarizability, radiative lifetimes, and dipole moments that can be precisely controlled via the principal quantum number $n$ [3]. Moreover, since the electron in a Rydberg atom is very weakly bound, such states can be ionized by relatively weak electric fields. In this case applied external fields can easily dominate the atomic Coulomb interaction, mimicking the conditions found in strong-field experiments and making it possible to test theories related to high-intensity regimes [10].

To put this into perspective, the (classical) electric field required to ionize atomic hydrogen is given by $F_{0} / 16 n^{4}$ [3], where the atomic unit of electric field for the $\mathrm{H}$ atom is $F_{0} \approx$ $5 \times 10^{9} \mathrm{~V} / \mathrm{cm}$ [11]. Thus, the fields required to ionize atomic hydrogen with $n=1,10$, and 20 are approximately $3 \times 10^{5}$, 30 , and $2 \mathrm{kV} / \mathrm{cm}$, respectively. As discussed in Sec. III, the ionization rates of Rydberg atoms depend not only on $n$ but also on the substructure within an $n$ manifold, meaning that selective field ionization (SFI) can be used for state-sensitive detection of highly excited atoms [12-14].

SFI is a technique that has been widely used in Rydberg experiments, including some of the first measurements of radiative lifetimes $[15,16]$, the effects of blackbody radiation [17,18], and collisional studies, including interactions between pairs of Rydberg atoms [19]. Here we describe experiments in which SFI has been used to study Rydberg

\footnotetext{
${ }^{1}$ Some exotic systems that can be thought of as molecules, such as molecular positronium ( $\mathrm{Ps}_{2}$ [1]), do not necessarily possess real Rydberg levels, although they may exhibit a Rydberg-like series of resonances [2].
}

positronium (Ps) atoms. In our experiments Ps atoms were allowed to fly into an ionization region in which either a static electric field was already present, or in which a pulsed electric field was applied after the atoms had entered. In both cases ionization was observed via the time dependence of the annihilation radiation generated by liberated positrons [20]. The electric fields in which Ps ionization occurred were measured for different $n$ states, including partial ionization of substates within $n$ manifolds [21], and found to be broadly consistent with calculated ionization rates [22] and Monte Carlo simulations, but with a slight disagreement observed for some lower- $n$ states. The discrepancy is small enough that it does not prevent the application of SFI methods to Ps experiments, but does nevertheless highlight the approximate nature of the calculations.

\section{EXPERIMENTAL METHODS}

\section{A. Ps production and excitation}

The experimental apparatus [23] as well the detection and data analysis methods [24] used in this work have been described elsewhere. A pulsed positron beam was generated using the moderated output of a ${ }^{22} \mathrm{Na}$ radioactive source [25] and a two-stage Surko-type buffer gas trap [26]. This system produced pulses containing $\sim 10^{5}$ positrons with Gaussian spatial (temporal) profiles approximately $2 \mathrm{~mm}(2 \mathrm{~ns})$ wide (full width at half maximum; FWHM) at a repetition rate of $1 \mathrm{~Hz}$. The positron beam was implanted in a porous silica film which produced Ps atoms with an efficiency of $\approx 30 \%$ [27]. The velocity distribution of ground-state Ps atoms emitted from the silica target in the direction of propagation of the beam may be represented by a Maxwell-Boltzmann distribution with $T=900 \mathrm{~K}$, and the angular distribution by a cosine function [20].

Ps atoms were optically excited using light generated by two pulsed dye lasers, employing the excitation scheme $1^{3} \mathrm{~S}_{1} \rightarrow 2{ }^{3} \mathrm{P}_{J} \rightarrow n^{3} \mathrm{D} / n^{3} \mathrm{~S}$. An ultraviolet (UV) laser $(\lambda=$ $243 \mathrm{~nm}$ ) with a fluence of $\sim 2 \mathrm{~mJ} / \mathrm{cm}^{2}$ and a bandwidth of $\Delta v=85 \mathrm{GHz}$ was used to drive $1{ }^{3} \mathrm{~S}_{1} \rightarrow 2{ }^{3} \mathrm{P}_{J}$ transitions. An infrared (IR) laser $(\lambda=740-733 \mathrm{~nm})$ was used to drive 


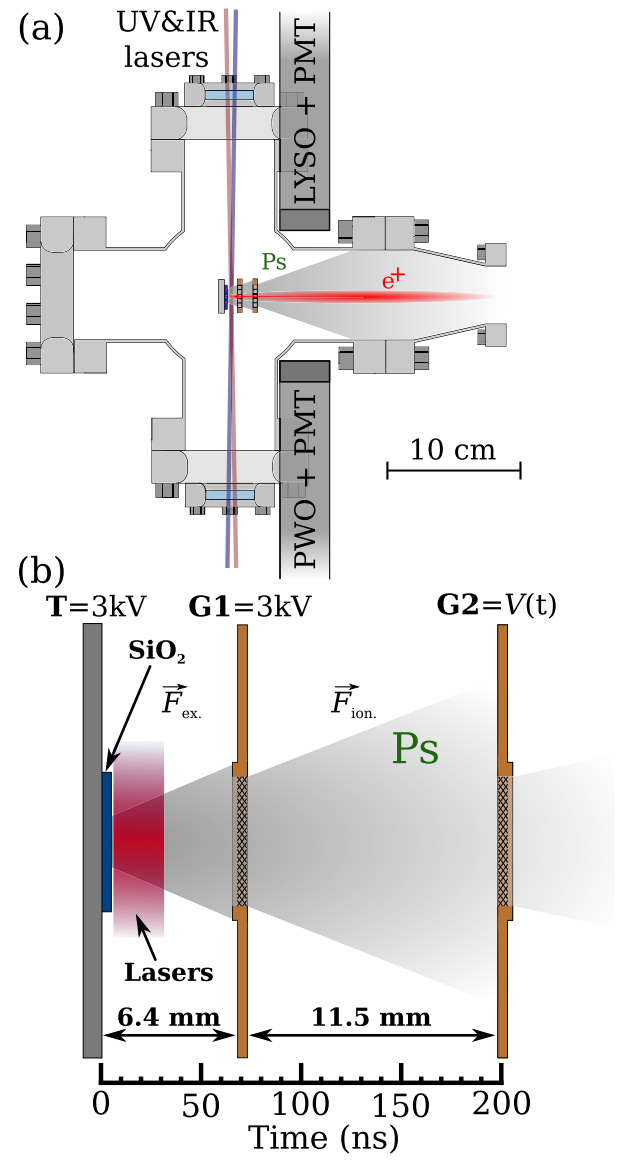

FIG. 1. Schematic of the Ps production chamber showing (a) the $\gamma$-ray detector positions and (b) Ps formation target and grid electrodes, indicating the positions of the excitation lasers and emitted Ps. The timescale indicates the Ps longitudinal flight time as determined from the mean speed of a $900 \mathrm{~K}$ Maxwell-Boltzmann distribution (see text).

$2{ }^{3} \mathrm{P}_{J} \rightarrow n^{3} \mathrm{D} /{ }^{3} \mathrm{~S}$ transitions, resulting in the production of Rydberg-Stark states with principal quantum numbers $n$ ranging from 18 to 25 . The IR laser fluence and bandwidth were $\sim 10 \mathrm{~mJ} / \mathrm{cm}^{2}$ and $5 \mathrm{GHz}$, respectively. The laser excitation arrangement is shown schematically in Fig. 1. Both lasers were linearly polarized in the vertical direction, perpendicular to the quantization axis, such that Rydberg atoms with azimuthal quantum number $m=0, \pm 2$ were produced [21].

Ps atoms were created in an electrode structure comprising two distinct regions (excitation and ionization) with independent electric fields, as shown in Fig. 1(b). For all of the measurements reported here the excitation region was operated with nominally zero electric field to avoid Stark broadening [21], while maintaining the required $3 \mathrm{kV}$ target bias [27]. The ionization region was operated with variable electric fields up to $2.5 \mathrm{kV} / \mathrm{cm}$, controlled via the bias applied to G2. The electric fields quoted in this work have an uncertainty on the order of $\pm 5 \%$ owing to variations in the flatness of the grids and electrodes. The grids used in the G1 and G2 electrodes were tungsten mesh with a 95\% open area.

Experiments were performed using both static and pulsed electric fields. In the latter case the G2 voltage was turned off using a fast high-voltage (HV) switch after Ps atoms

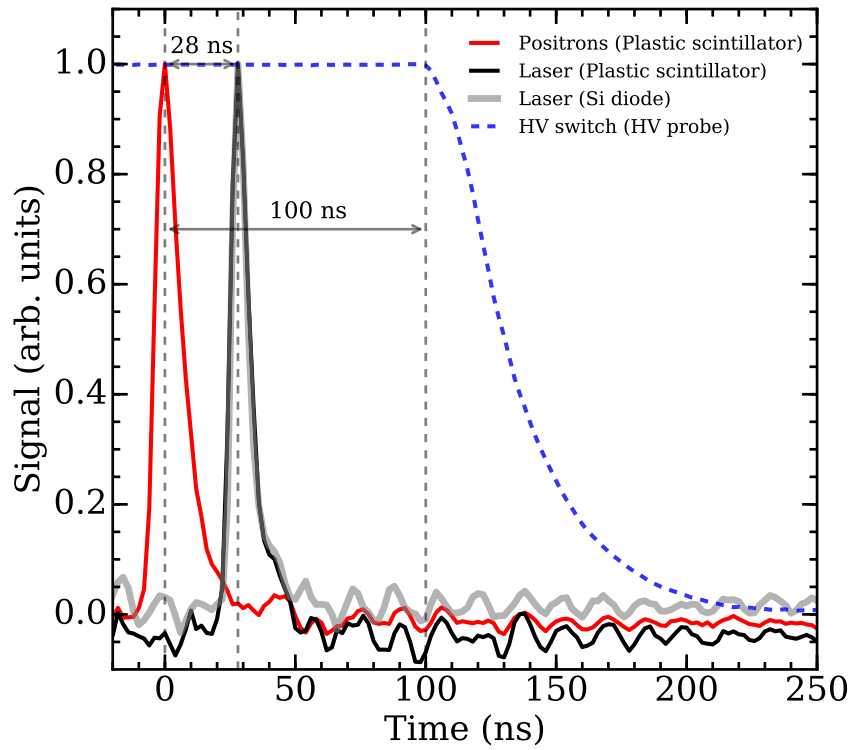

FIG. 2. Relative timing of positron and laser pulses and highvoltage (HV) switch. The positron implantation time was measured via annihilation radiation by using a plastic scintillator coupled to a PMT. This detector has a pinhole to admit laser light allowing the laser pulse arrival time to be measured also. A fast Si photodiode detector was used to measure the laser pulse arrival time relative to the HV switch voltage using a fast HV probe. All data are amplitude normalized; the $\mathrm{HV}$ signal is switching from $2.5 \mathrm{kV}$ to $0 \mathrm{~V}$.

had entered the ionization region (see Fig. 2). The time between Ps production and field switching was determined from measurements shown in Fig. 2. A plastic scintillator and photomultiplier tube (PMT) were used to directly measure the time interval between the positron and laser pulses [28], and a fast silicon diode detector was used to measure the true arrival time of the laser pulse. This measurement was then compared with a fast HV probe signal to obtain the interval between the positron implantation and the high-voltage switching, accounting for the intrinsic PMT delay. The switching times were optimized experimentally, but must be characterized in this way to be properly included in simulations and to extract electric fields from data recorded in the time domain.

\section{B. $\gamma$-ray spectroscopy}

Ps production was monitored using single-shot positron annihilation lifetime spectroscopy (SSPALS) [29]. Here Ps annihilation $\gamma$ radiation is detected by a scintillator coupled to a PMT. Detectors are placed outside the vacuum chamber near the Ps production region as indicated in Fig. 1(a). In this work two different scintillators were used, lutetium-yttrium oxyorthosilicate (LYSO) [24] and lead tungstate (PWO) [30]. These scintillators have decay times of approx 40 and $10 \mathrm{~ns}$, respectively. LYSO is more efficient than PWO and is generally used when timing information is not critical. Unless otherwise specified, all data presented here were recorded using a LYSO-based detector.

Examples of single-shot lifetime spectra recorded with a LYSO detector are shown in Fig. 3(a). For these measurements atoms have been excited and photoionized; the resulting spectra differ because the amount of long-lived Ps is reduced 


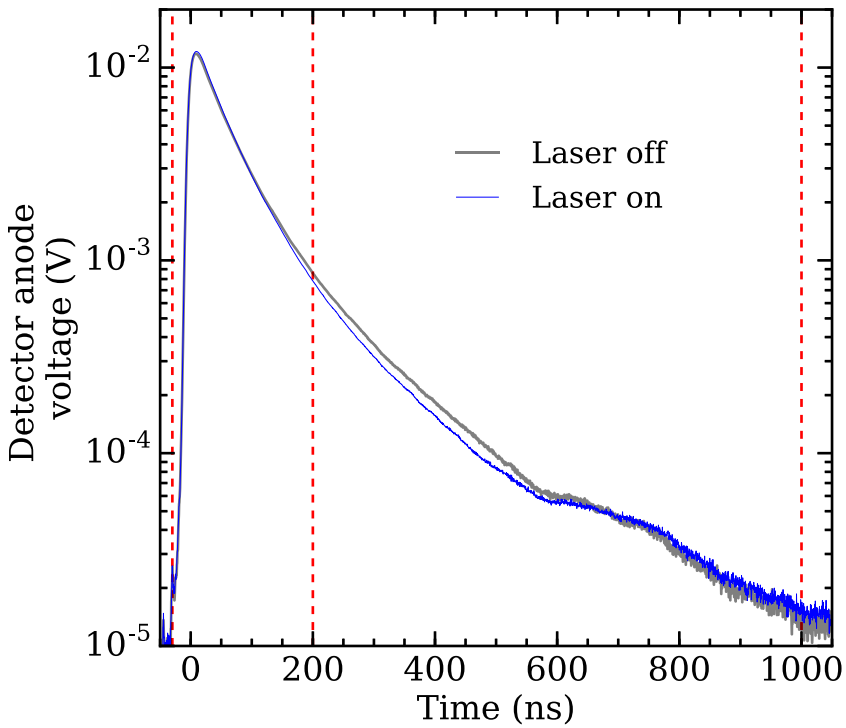

FIG. 3. SSPALS spectra measured by using a LYSO scintillator with and without the presence of UV laser light tuned to drive $1{ }^{3} \mathrm{~S}_{1} \rightarrow 2{ }^{3} \mathrm{P}_{J}$ transitions $(\lambda=243 \mathrm{~nm})$ and an ionizing IR laser $(\lambda=729 \mathrm{~nm})$. The vertical dashed lines indicate the time windows used to determine the indicated $f$ values as described in the text.

following ionization. These data are parametrized by the quantity $f$, which is defined as

$$
f=\int_{B}^{C} V(t) d t / \int_{A}^{C} V(t) d t
$$

where $V(t)$ is the PMT output voltage and $A, B$, and $C$ are integration time windows. The vertical lines in Fig. 3 indicate the analysis time windows used throughout this work, namely $(A, B, C)=(-30,200,1000)$ ns. Data were recorded for two cases; namely, with the excitation lasers tuned on (Sig) and off (Back) resonance with the $1{ }^{3} \mathrm{~S}_{1} \rightarrow 2{ }^{3} \mathrm{P}_{J}$ transition, as well as an ionizing IR laser pulse. Laser-induced effects are characterized by the parameter $S_{\gamma}$, defined as

$$
S_{\gamma}=\frac{\left(f_{\text {Back }}-f_{\text {Sig }}\right)}{f_{\text {Back }}} .
$$

The difference curve between the laser on and off spectra in Fig. 3 is shown in Fig. 4(a). These data indicate the time profile of Ps annihilation events relative to the decay of ground-state atoms. Thus, Fig. 4(a) exhibits an excess peak at early times due to laser-induced ionization events, followed by a reduction in annihilation events indicated by the negative signal. Other processes can also be identified in this way, as shown in Figs. 4(b) and 4(c). These figures show similar data obtained following the excitation of $n=20$ Ps atoms with and without an ionizing electric field present, respectively. The data in Fig. 4(b) are similar to the ionization data in Fig. 4(a), except that the peak is delayed and broadened because of the time Ps atoms take to reach the ionization region. In Fig. 4(c), Ps atoms have lifetimes of more than $20 \mu \mathrm{s}$ [31], and the observed annihilation events are caused by collisions with electrodes or the vacuum chamber walls. Thus, the delayed excess annihilation events are spread out in time, with a peak at $\approx 200 \mathrm{~ns}$, corresponding to atoms that

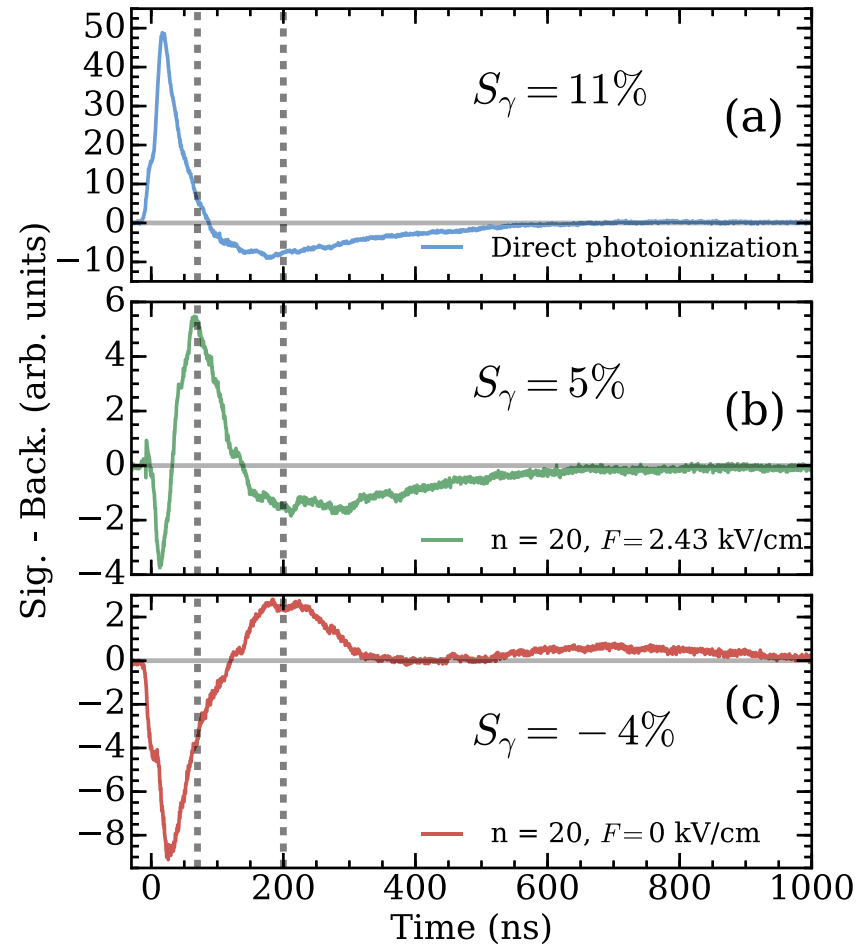

FIG. 4. Background subtracted spectra for (a) Direct IR photoionization, (b) excitation of $n=20$ with subsequent field ionization and, (c) excitation of $n=20$ without field ionization. The electric fields present in the ionization region are indicated in panels (b) and (c). The dashed lines indicate the times at which Ps atoms will reach G1 and G2 as determined by the mean speeds of the Maxwell-Boltzmann distribution used to represent the Ps velocity (see text). Also shown are the $S_{\gamma}$ values obtained using Eq. (2).

collide with the second grid electrode G2 [see Fig. 1(b)]. Note that, with the analysis time windows used, decay events that happen early (late) relative to $B$ (i.e., $200 \mathrm{ns)} \mathrm{will} \mathrm{result} \mathrm{in}$ positive (negative) $S_{\gamma}$ values, as indicated in Fig. 4.

\section{THEORETICAL BACKGROUND}

In the presence of an electric field, $\vec{F}=(0,0, F)$, the Hamiltonian for the Ps atom, neglecting spin-spin and spinorbit contributions, can be expressed as

$$
\begin{aligned}
H & =-\frac{\hbar^{2}}{2 \mu_{\mathrm{Ps}}} \nabla^{2}-\frac{e^{2}}{4 \pi \epsilon_{0} r}+e \vec{F} \cdot \vec{r} \\
& =-\frac{\hbar^{2}}{2 \mu_{\mathrm{Ps}}} \nabla^{2}-\frac{e^{2}}{4 \pi \epsilon_{0} r}+e F z,
\end{aligned}
$$

where $\mu_{\mathrm{Ps}}=m_{\mathrm{e}^{+}} m_{\mathrm{e}^{-}} /\left(m_{\mathrm{e}^{+}}+m_{\mathrm{e}^{-}}\right), \vec{r}=(x, y, z)$ is the radial position vector of magnitude $r=|\vec{r}|$, and $\hbar, e$, and $\epsilon_{0}$ are the reduced Planck constant, the electron charge and the vacuum permittivity, respectively.

The third term in Eq. (4) breaks the spherical symmetry of the Hamiltonian and hence the corresponding Schrödinger equation. In this situation the potential-energy contributions to the Hamiltonian remain dependent on $-1 / x$ and $-1 / y$ in the directions perpendicular to the electric field. However, as can be seen in Fig. 5(a) for the case in which $F=850 \mathrm{~V} / \mathrm{cm}$, 

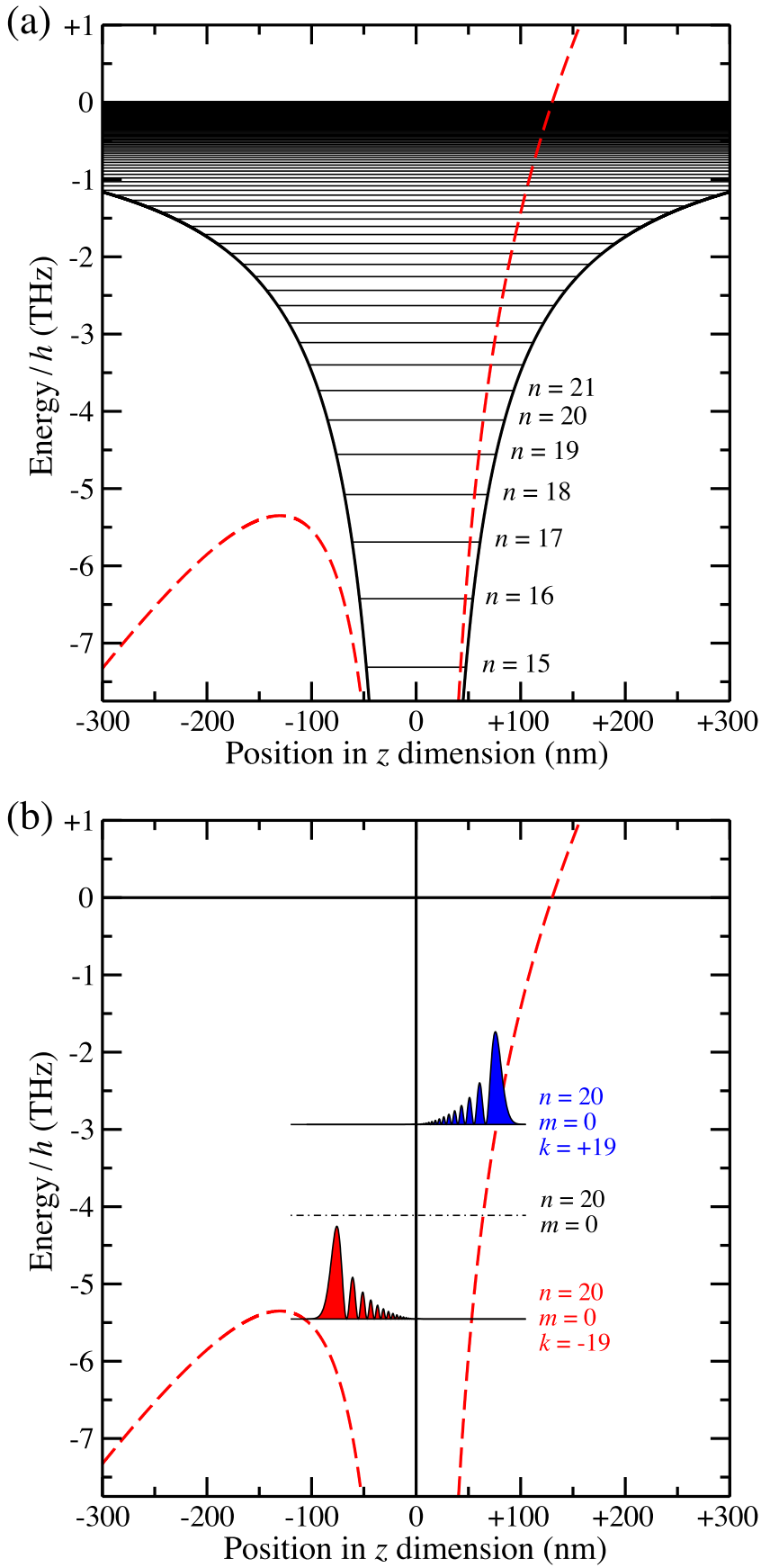

FIG. 5. (a) Comparison of the pure Coulomb potential of the Ps atom in the absence of external fields (continuous black curve) with the potential in the direction of an applied electric field of $F=850 \mathrm{~V} / \mathrm{cm}$ (dashed red curve). The field-free Rydberg level energies for $n \geqslant 15$ in the pure Coulomb potential are indicated by the horizontal lines. (b) The energies and radial charge distributions of the outermost Stark-shifted $n=20$ sublevels with $m=0$ and $k= \pm 19$ in the potential when $F=850 \mathrm{~V} / \mathrm{cm}$ (dashed red curve). For reference the energies of the field-free $n=20$ levels with $m=0$ are indicated by the horizontal dash-dotted line.

the potential in the direction of the field (dashed red curve) deviates significantly from that of a pure Coulomb potential (continuous black curve). The saddle point at $z=-130 \mathrm{~nm}$ in the potential including the electric-field term in Fig. 5(a) gives rise to two classes of eigenstates. These include the states which lie above this Stark saddle point and are directly coupled to the ionization continuum, and the states that lie below the saddle point and tunnel slowly through the barrier to ionize. Comparison of the saddle-point energy in Fig. 5(a) with the field-free Rydberg level energies (horizontal black lines) indicates that, in the case depicted, these states are the set for which $n \geqslant 18$, and the set for which $n<18$, respectively. From the dependence of the position of the saddle point in Fig. 5(a) on the strength of the electric field, the "classical" ionization electric field-for which the eigenenergy of a state with principal quantum number $n$ exceeds that of the saddle point-can be expressed as $F_{\text {Class }}=F_{\mathrm{Ps}} /\left(16 n^{4}\right)$, where $F_{\mathrm{Ps}}=2 h c R_{\mathrm{Ps}} /\left(e a_{\mathrm{Ps}}\right)=1.286 \times 10^{9} \mathrm{~V} / \mathrm{cm} \mathrm{[3]}$.

For single-electron systems such as hydrogen or positronium in an electric field, the Schrödinger equation, although not separable in spherical polar coordinates, remains separable in parabolic coordinates. The resulting eigenstates are denoted by the parabolic quantum numbers $n_{1}$ and $n_{2}$. The static electric-dipole moments, and hence the Stark energy shifts, of these states are dependent on $k=n_{1}-n_{2}$. For a particular value of $n$ and azimuthal quantum number $m$, the allowed values of $k$ range from $-(n-|m|-1)$ to $+(n-|m|-1)$ in intervals of two. The energy shift of each $k$ state in an electric field $F$ can then be expressed (to third order) as [22]

$$
\begin{aligned}
E_{\text {Stark }}= & \frac{3}{2} n k e a_{0_{\mathrm{Ps}}} F \\
& -\frac{1}{16} n^{4}\left(17 n^{2}-3 k^{2}-9 m^{2}+19\right) \frac{e^{2} a_{0_{\mathrm{Ps}}}^{2}}{E_{\mathrm{h}_{\mathrm{Ps}}}} F^{2} \\
& +\frac{3}{32} n^{7} k\left[23 n^{2}-k^{2}+11 m^{2}+39\right] \frac{e^{3} a_{0_{\mathrm{Ps}}^{3}}^{3}}{E_{\mathrm{h}_{\mathrm{Ps}}}^{2}} F^{3},
\end{aligned}
$$

where $E_{\mathrm{h}_{\mathrm{ps}}}=2 h c R_{\mathrm{Ps}}$. The electric field does not couple sublevels with different values of $m$. Because the parabolic eigenfunctions represent a set of stationary states in the field, in the limit as $F \rightarrow 0$ the Stark energy shift of each of these states is predominantly linear. In high fields, the second and thirdorder contributions begin to play an increasingly significant role with the onset of $n$ mixing. This can be seen in Fig. 6 for the case of the manifold of Stark states with $n=20$ and $m=0$.

The first term in Eq. (5) represents the first-order interaction of a static electric dipole with an electric field. This interaction can be expressed in general as $E_{\text {static }}=$ $-\vec{\mu}_{\text {elec }} \cdot \vec{F}$. Therefore, the static electric-dipole moment of each of the Stark states described by Eq. (5) is $\vec{\mu}_{\text {elec }}=$ $\left[0,0,-(3 / 2) n k e a_{0_{\mathrm{Ps}}}\right]$. For values of $k<0, \vec{\mu}_{\text {elec }}$ lies parallel to $\vec{F}$. Consequently, the reduced electron charge is located on the same side of center of mass of the system as the Stark saddle point in Fig. 5(a). On the other hand, for values of $k>0, \vec{\mu}$ lies antiparallel to $\vec{F}$ and the charge is located on the opposite side of the center of mass to the saddle point. This can be seen in Fig. 5(b) where the charge distributions in the direction of the electric field are displayed for the outermost $n=20$ Stark states for which $m=0$ and $k= \pm 19$. The eigenenergies of these two Stark states in the field are 


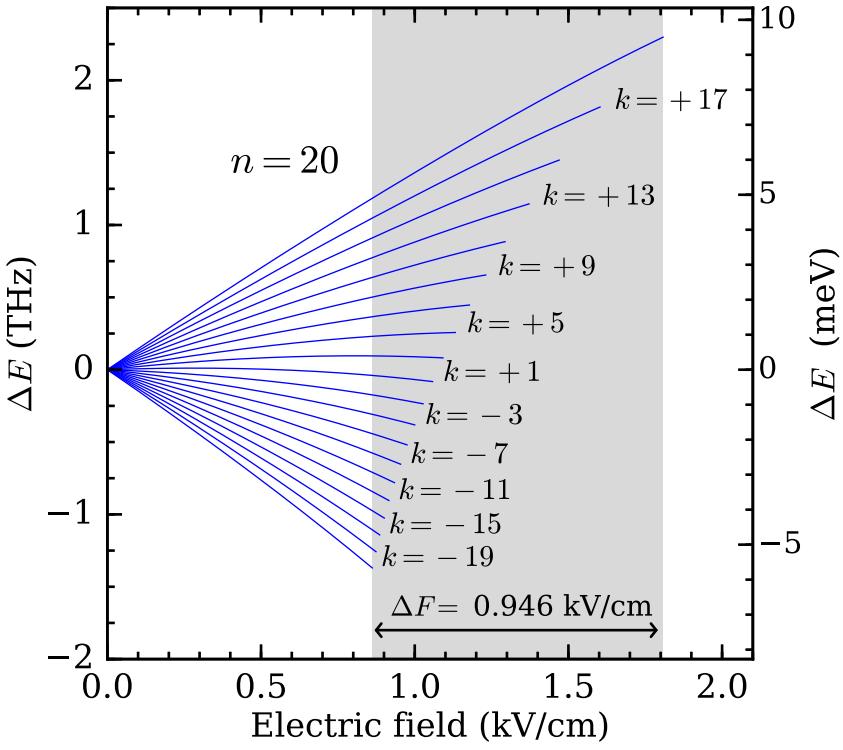

FIG. 6. Energy-level diagram of the Stark splitting of the $m=0$ manifold of $n=20$. The curves corresponding to states labeled by $k$ are truncated at the electric field that leads to ionization rates of $7.7 \mathrm{MHz}$, the inverse of the average Ps transit time through the ionization region (see Sec. IV).

indicated by the vertical offset of each charge distribution. Because of their negative (positive) Stark energy shifts, and hence the direction of the force exerted on them in an inhomogeneous electric field, states with $k<0(k>0)$ are referred to as high-field-seeking (HFS) [low-field-seeking (LFS)] states, respectively. For reference, the energy of the $n=20$ Rydberg levels with $m=0$ in the absence of the field are indicated by the horizontal dashed line in Fig. 5(b).

The orientation of the static electric-dipole moments of the Stark states affects their electric-field ionization dynamics through the sign of their first-order Stark shifts, and their propensity to tunnel ionize. Accounting for the first-order Stark energy shift of the outer $k=-(n-|m|-1)$ state, in which the reduced electron charge is located close to the Stark saddle point [e.g., lower red charge distribution in Fig. 5(b)], the "classical" ionization electric field becomes $F_{\text {Class }}=$ $F_{\mathrm{Ps}} /\left(9 n^{4}\right)$. In the case of the outer $k=+(n-|m|-1)$ state, the charge remains localized on the opposite side of the center of mass of the system with respect to the Stark saddle point [e.g., upper blue charge distribution in Fig. 5(b)] when only the first-order contribution from the field is considered. However, for fields in which ionization becomes important the higher-order contributions to the Stark energy shift depolarize the atom redistributing the charge density in states with $k>0$ toward the side of the center of mass close to the Stark saddle point. As this occurs ionization becomes increasingly likely and for the outer $k=+(n-$ $|m|-1)$ state this occurs at a field approximately twice as large as that required to ionize the outer $k=-(n-|m|-1)$ state.

A more complete treatment of the electric-field ionization of these hydrogenic Rydberg-Stark states requires the calculation of their tunnel ionization rates, $\Gamma_{n, n_{1}, n_{2}, m}(F)$. This can be achieved using the semi-empirical expression of

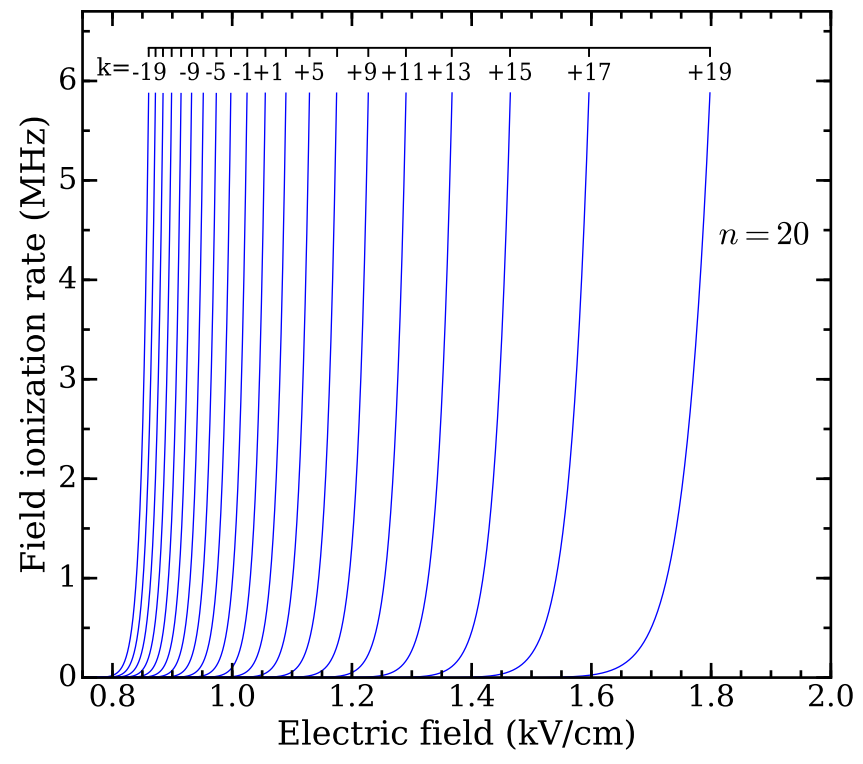

FIG. 7. Field-ionization rates for the $n=20$ manifold of Stark states as a function of electric field. The curves are terminated at ionization rates of $7.7 \mathrm{MHz}$, the inverse of the average Ps transit time through the ionization region in the experiments (see Sec. IV).

Ref. [22]:

$$
\begin{aligned}
\Gamma_{n n_{1} n_{2} m}= & \frac{E_{\mathrm{h}_{\mathrm{p}}}}{\hbar} \frac{(4 R)^{2 n_{2}+m+1}}{n^{3} n_{2} !\left(n_{2}+m\right) !} \exp \left[-\frac{2}{3} C-\frac{1}{4} n^{3} \frac{e a_{0_{\mathrm{Ps}}} F}{E_{\mathrm{h}_{\mathrm{s}}}}\right. \\
& \left.\times\left(34 n_{2}^{2}+34 n_{2} m+46 n_{2}+7 m^{2}+23 m+\frac{53}{3}\right)\right]
\end{aligned}
$$

where

$$
C=\frac{1}{e a_{0 \mathrm{ps}} \sqrt{E_{\mathrm{h}_{\mathrm{Ps}}}}} \frac{\left(-2 E_{n n_{1} n_{2} m}\right)^{3 / 2}}{F},
$$

and $E_{n n_{1} n_{2} m}=-\left[E_{\mathrm{h}_{\mathrm{Ps}}} /\left(2 n^{2}\right)\right]+E_{\mathrm{Stark}}$, is the total energy of the state, with respect to the ionization limit, in the presence of the electric field. The tunnel ionization rate of the outermost $k=-(n-|m|-1)$ Stark state in the classical ionization field is approximately $10^{8} \mathrm{~s}^{-1}$. A similar rate is achieved for the outermost $k=+(n-|m|-1)$ Stark state when the field is twice as large.

The ionization rates calculated using this approach for the $n=20$ Stark states of Ps with $m=0$ are displayed in Fig. 7 . It can be seen from these data that, upon reaching a certain limit, the ionization rates increase exponentially as the electric field is further increased. For example, for $n=20$ as depicted in Fig. 7, the field-ionization rate scaling with the electric field $F$ range between $\Gamma \propto F^{30}$ for $k=+19$ and $\Gamma \propto F^{90}$ for $k=-19$. Because of this, the finite timing resolution in the measurements reported here means that it is not possible to distinguish these individual rates. Instead the gradual onset of ionization across the manifold of Stark states associated with each value of $n$ studied is seen. In the experiments the nominal tunnel ionization rates of interest correspond to the inverse of the average transit time $(7.7 \mathrm{MHz})$ of the Ps atoms through the ionization region between G1 and G1 in Fig. 1. 
The range of electric fields over which this rate is reached for the $n=20$ Rydberg-Stark states with $m=0$ can be seen in Fig. 6 in which the curve representing each state is truncated at this value.

As discussed in Ref. [22], the semi-empirical expression in Eq. (6) is most accurate for low values of $m$ in weak fields. The corresponding rates deviate from the true ionization rates for the outermost Stark states, i.e., those for which $k= \pm(n-|m|-1)$. Particularly in fields for which the onset of ionization on experimentally significant timescales occurs, i.e., for $\Gamma>1 \mathrm{MHz}$. For the states considered in Ref. [22] with values of $n<30$ deviations of up to a factor of five from exact numerical results were identified in the ionization rates calculated by using Eq. (6).

\section{SIMULATIONS}

Monte Carlo simulations of Ps field ionization were carried out by using the tunnel ionization rates obtained from Eq. (6), as discussed in Sec. III. In the case of experiments performed in static electric fields the measured ionization signal depends strongly on the geometry of the electrode structure and the Ps velocity distribution. These were therefore included in the simulation. The Ps velocity was represented by a MaxwellBoltzmann distribution $(T=900 \mathrm{~K})$ and the corresponding angular distribution by a cosine function. Although Ps emission from porous silica films is not expected to be thermal, previous measurements [20] indicate that the true distribution can be represented in this way by artificially adjusting the distribution temperature.

The velocity distribution of the subset of atoms excited to Rydberg states was determined by applying threshold conditions for the spatial, temporal, and spectral overlap of a $\Delta t=5 \mathrm{~ns}$ pulsed laser field with the ground state Ps atoms. The spatial overlap was based on a $4 \times 8 \mathrm{~mm}$ laser beam. The spectral overlap was determined by the UV laser bandwidth $(\Delta v=100 \mathrm{GHz})$ and the Doppler shift of each atom as a result of the component of its velocity parallel to the direction of UV laser propagation. Rydberg atoms were produced with equal populations in the $m=0$ and $|m|=2$ manifolds, as dictated in the experiment by the polarization of the laser light used (see Sec. II A). Each atom was assigned a random value of $k$ from the allowed range specified in Sec. III.

The equations of motion of each atom were solved to obtain their trajectories by using the fourth-order RungeKutta algorithm, where the acceleration of each atom due to the electric-field gradients in the apparatus is given by

$$
\vec{a}=\frac{\mu_{\mathrm{elec}}}{2 m_{e}} \nabla|\vec{F}|
$$

where $\mu_{\text {elec }}$ is the static electric-dipole moment associated with the respective Rydberg-Stark state and the electric-field gradients were obtained through finite-element calculations of the electric field within the apparatus.

In the trajectory simulations, the time dependence of annihilation counts was determined based on decay events due to (1) tunneling ionization given by Eq. (6), and subsequent positron annihilation, and (2) Ps collisions with the chamber walls and electrodes. Radiative decay from Rydberg states was negligible. Lifetime spectra were generated by the

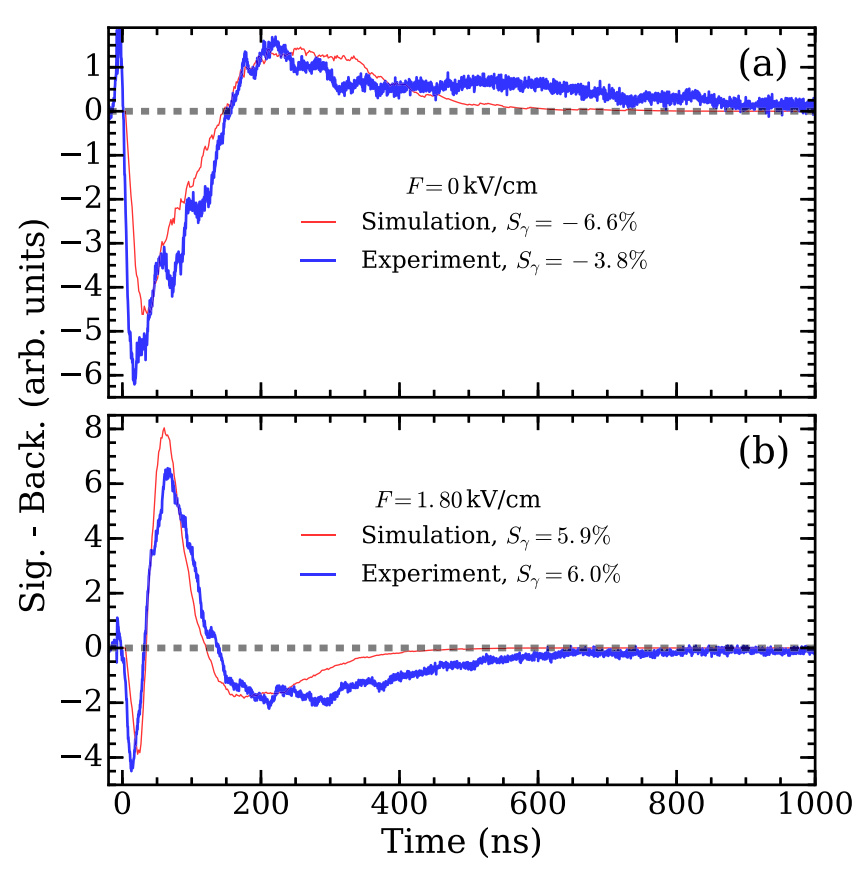

FIG. 8. Comparison between measured and simulated difference spectra similar to those shown in Fig. 4 for $n=20$ Ps with (a) zero electric field, and (b) a static electric field of $\approx 1.8 \mathrm{kV} / \mathrm{cm}$ in the ionization region. These fields will result in no ionization and complete ionization, respectively.

convolution of the annihilation signal with the detector response function, which is an exponential decay with $t_{\text {det }}=$ $40 \mathrm{~ns}$ [24]). Subtracting a simulated ground-state lifetime spectrum gives the background-subtracted signal that can be compared directly with measurements.

The simulations were run using 3000 Ps atoms propagating for $1200 \mathrm{~ns}$. A comparison between measured and simulated background-subtracted signals for $n=20$ Ps in two different cases is shown in Fig. 8. These cases are (a) production of long-lived atoms with no ionization [Fig. 8(a)] and (b) production of $n=20$ atoms in a fully ionizing electric field of $1.8 \mathrm{kV} / \mathrm{cm}$ [Fig. 8(b)]. We find broad agreement in the two cases which is improved after adjusting the MaxwellBoltzmann distribution used to represent the Ps energy.

Simulations were also performed for the case when the ionizing electric field was switched on after the Rydberg Ps atoms had entered the ionization region. Since in this configuration all of the Ps atoms experience the same electric field at the same time, these simulations did not require inclusion of any geometrical parameters or trajectories. These simulations were initialized with $10^{6}$ Ps atoms in a specific $n$ state. A time-dependent electric field (see Fig. 2) was generated for each $0.4 \mathrm{~ns}$ time step $\Delta t$. A Monte Carlo algorithm was used to determine the number of Ps atoms that decayed at each time step, and an ionization rate was calculated for each atom using Eq. (6). The resulting spectrum was then convoluted with the detector response for comparison with the experimental data.

The measured relative times between positron implantation, laser excitation, and the potential applied to electrode G2 are shown in Fig. 2. These data are included explicitly in the simulation so that the electric field experienced by different 
atoms as a function of time can be represented. Other than scaling the laser off $f$ values to account for the prompt Ps annihilation peak, the Monte Carlo simulations contain no free parameters and are expected to be both qualitatively and quantitatively comparable to the measured data [32].

\section{RESULTS AND DISCUSSION}

\section{A. Static field ionization}

Experiments were performed using both static and pulsed electric fields. In the static field configuration a dc voltage of $3 \mathrm{kV}$ was applied to the target and to electrode $\mathrm{G} 1$, with the $\mathrm{G} 2$ voltage varied [see Fig. 1(b)]. The incident positron beam was unaffected by the applied electric fields and was implanted into the silica target with an energy of $3 \mathrm{keV}$. Ps atoms emitted from the target were excited to Rydberg states and were able to fly into the ionization region at a rate determined by the electrode geometry and the Ps velocity distribution, as described in Sec. IV.

A spectrum of $2{ }^{3} \mathrm{P}_{J} \rightarrow n^{3} \mathrm{D} / n^{3} \mathrm{~S}$ transitions, with $n$ ranging from 17 to 26 , is shown in Fig. 9. These data were recorded using a static ionization field of $2.5 \mathrm{kV} / \mathrm{cm}$, thus generating positive $S_{\gamma}$ values (see Sec. II). The approximately equal height of the peaks implies that the transitions to Rydberg levels were saturated. These data cover the range of $n$ over which the experiments reported here were conducted. Although it was possible to excite higher $n$ states, our resolution was limited by the laser bandwidth and Doppler broadening [21], as is evident from the overlap of the spectral features associated with the transitions to states for which $n \geqslant 25$ in Fig. 9. The production of lower $n$ states was also possible, but the electric fields required to ionize the atoms becomes prohibitively large. All of the Rydberg states studied here have lifetimes longer than $15 \mu \mathrm{s}$ [31] so that radiative Ps decay was entirely negligible.

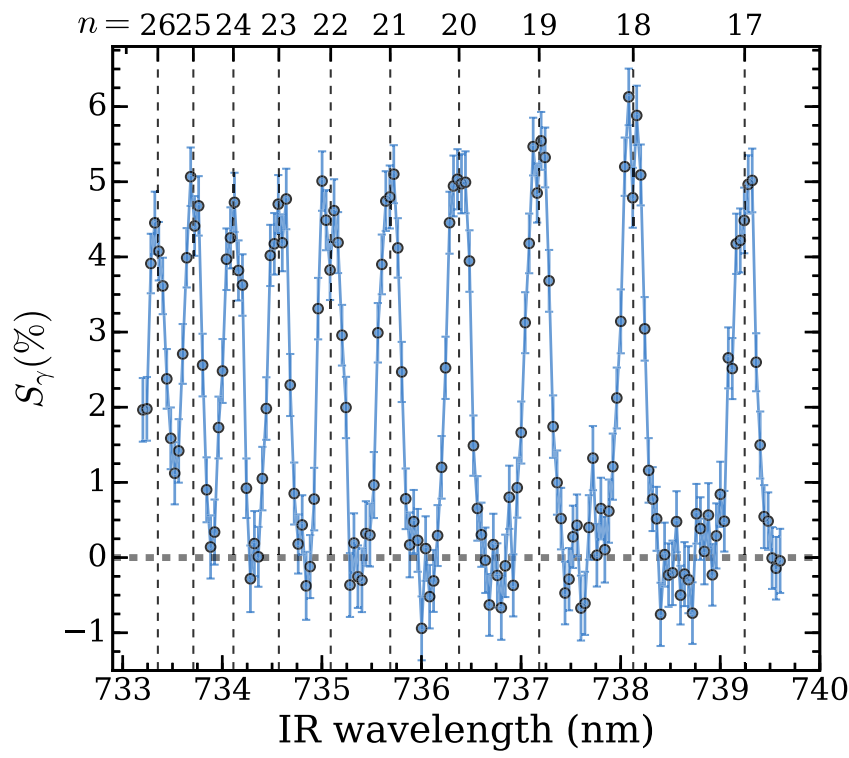

FIG. 9. Excitation spectrum for $2{ }^{3} \mathrm{P}_{J} \rightarrow n^{3} \mathrm{D} / n^{3} \mathrm{~S}$ transitions, with $n$ ranging from 17 to 26 . These data were recorded with an ionization field of $2.5 \mathrm{kV} / \mathrm{cm}$. The dashed vertical lines indicate the expected transition wavelengths.

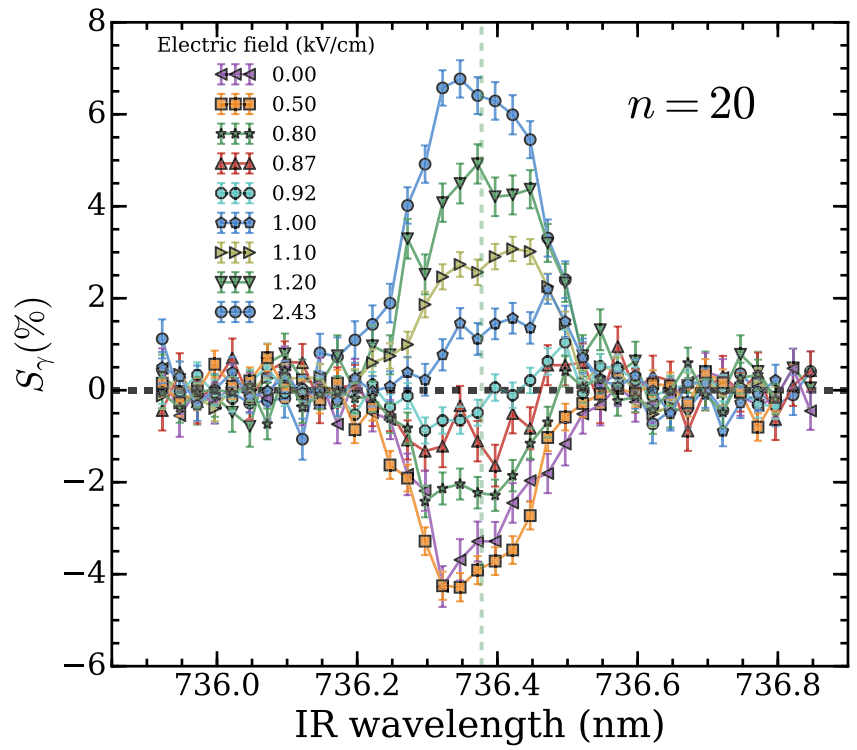

FIG. 10. $2{ }^{3} \mathrm{P}_{J} \rightarrow 20{ }^{3} \mathrm{D} / 20{ }^{3} \mathrm{~S}$ transitions measured at various ionization electric fields as shown in the legend. The dashed vertical line indicates the expected field-free excitation wavelength.

Figure 10 shows spectra of $2{ }^{3} \mathrm{P}_{J} \rightarrow 20^{3} \mathrm{D} / 20^{3} \mathrm{~S}$ transitions recorded with a range of electric fields applied in the ionization region. Because the transitions are saturated and the atoms are excited in zero electric field, we expect to populate all of the accessible $k$ states approximately equally. Then the value of $S_{\gamma}$ in an electric field where half of the atoms are ionized will be approximately zero, and will not depend on the laser wavelength, as observed in Fig. 10. Here the line intensity exhibits complete inversion as the fields are switched from fully ionizing to nonionizing, with a nearly flat region in between. A small asymmetry is observed for the $0.92 \mathrm{kV} / \mathrm{cm}$ field, indicating that there may be some selective population of $k$ states when the laser is tuned to the edge of the lineshape [21].

Figure 11(a) shows $S_{\gamma}$ as a function of the static electric field for various $n$ states. At low fields $S_{\gamma}$ is negative, indicating that no ionization has taken place. As the field is increased $S_{\gamma}$ changes sign, passing through zero, as more atoms are ionized (cf. Fig. 10). The fields required to accomplish this increase with $n$, as expected. The shape of these curves is related to the variation in the ionization fields for different $k$ states (see Fig. 6). The point at which $S_{\gamma}=0$ roughly corresponds to the field at which all of the HFS states have been ionized. That is, when approximately half of the Ps atoms have been ionized the positive and negative parts of the integrated lifetime spectra are comparable and thus $S_{\gamma}$ is close to zero.

In both Figs. 10 and 11(a), the maximum and minimum magnitudes of $S_{\gamma}$ are not the same. This is because, in the ionizing case (positive $S_{\gamma}$ ), all atoms are detected via annihilation radiation originating from the same location, whereas in the nonionizing case (negative $S_{\gamma}$ ) the atoms are detected only after collisions with chamber walls and other material objects in the vacuum system (see also Fig. 4). This changes the detection efficiency and affects the magnitude of $S_{\gamma}$. Owing to their long lifetimes some atoms fly out of the detection 


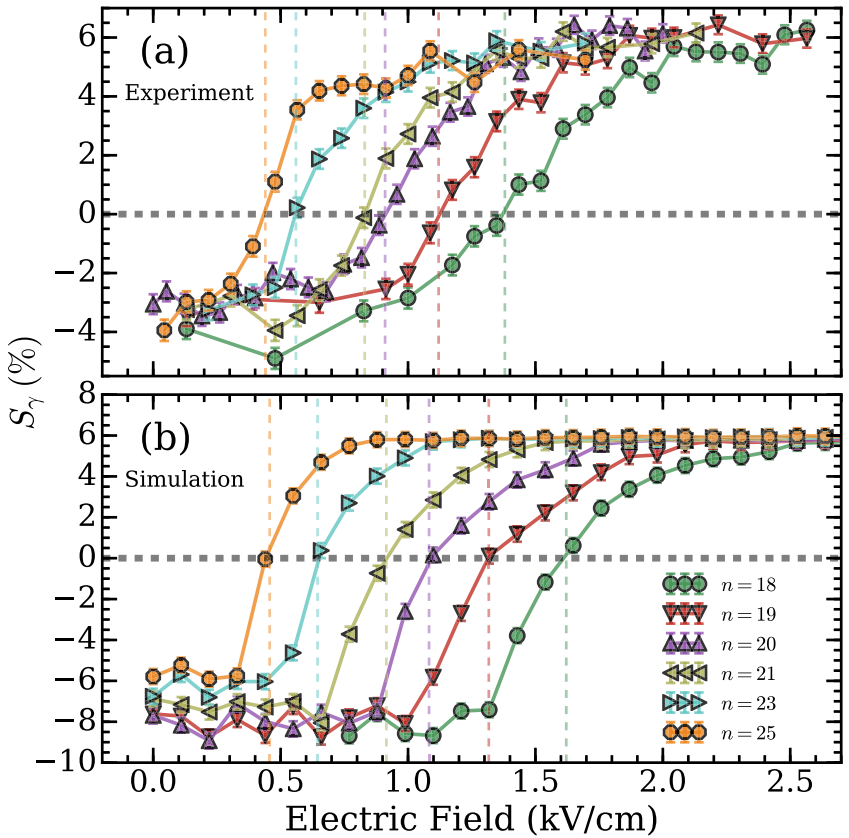

FIG. 11. (a) $S_{\gamma}$ as a function of the static electric field in the ionization region for Rydberg Ps atoms with $n=18,19,20,21,23$, and 25. (b) Monte Carlo simulations corresponding to the measurements. The dashed vertical lines indicate the fields at which $S_{\gamma}=0$ for the different states.

region and are never observed. This effect is represented in the simulations, giving rise to an asymmetry between the magnitudes of positive and negative $S_{\gamma}$ values. However, it is not identical to the measured asymmetry because the detection efficiency for very long lived atoms is not well known and is not included in the simulation. It is possible to adjust the analysis time windows to compensate for this; however, doing so has only a small effect on the fields for which $S_{\gamma}$ crosses through zero. Therefore these windows have not been adjusted in the analysis presented here.

The observed $S_{\gamma}=0$ fields obtained from the measured and simulated data shown in Fig. 11(a) are plotted in Fig. 12, along with calculated fields for LFS, HFS, and $k=0$ Stark states, using an ionization rate given by the inverse of the mean Ps flight time through the ionization region $(7.7 \mathrm{MHz})$. This figure highlights the fact that the ionization fields are not the same for different Stark states, and the dependence on the value of $n$ does not scale exactly as $1 / n^{4}$. This deviation from exact $1 / n^{4}$ scaling is caused by the contribution of the high-order terms in Eq. (5) to the ionization rate [see Eq. (6)] and can also be seen from the asymmetry in the ionization fields in the Stark map in Fig. 6. The electric fields required to ionize HFS states are lower than those required to ionize LFS states at the same rate by approximately a factor of two, as discussed in Sec. III.

The simulated $S_{\gamma}=0$ data agree well with the calculated $k=0$ fields (which are equivalent to the average between $k= \pm 1)$ as they must, since the ionization rates are built into the simulations. This agreement does, however, serve as a validation of the velocity and angular distributions included in the simulations. There is a clear discrepancy between the measured $k=0$ ionization fields and those obtained from the

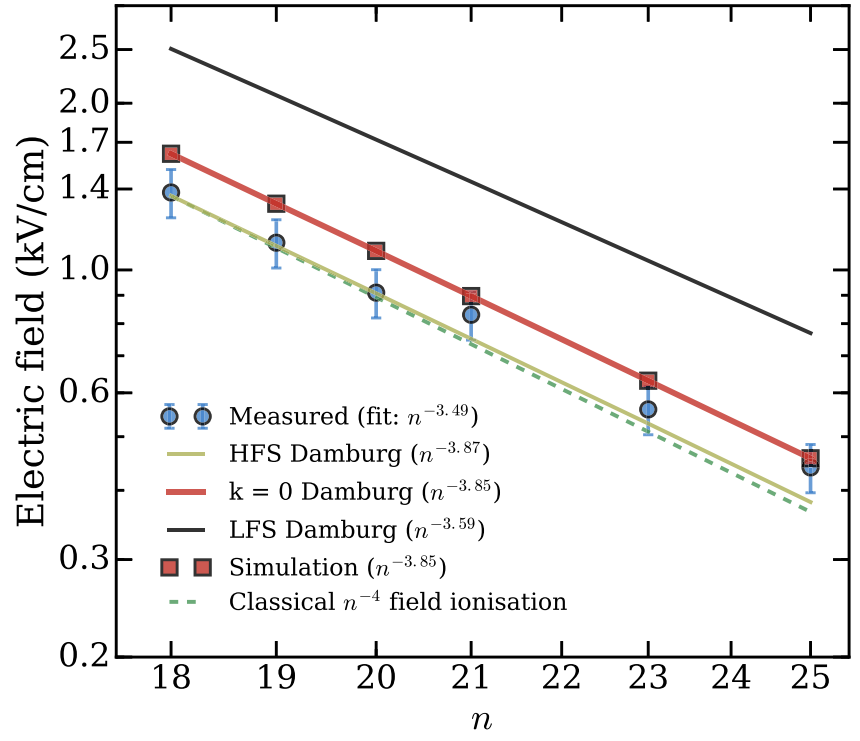

FIG. 12. Measured and simulated electric fields at which $S_{\gamma}=0$. Also shown is the classical $1 / n^{4}$ ionization field dependence, and the fields at which HFS, LFS, and $k=0$ Stark states ionize at $7.7 \mathrm{MHz}$ as determined from Eq. (6). The exponents of the $n$ dependencies are indicated in the figure, including that obtained from a fit to the data and the simulations.

calculations; the agreement is worse for lower $n$ states, as is also apparent from the data shown in Fig. 11. The complete explanation for this is not presently known but appears to be simply due to the limitations of the semi-empirical approach used in the calculations [22].

\section{B. Pulsed-field ionization}

Measurements were also performed using pulsed electric fields. In these experiments Ps atoms were allowed to fly into the ionization region (see Fig. 1) with $3 \mathrm{kV}$ applied to both electrode G2 and G1 (i.e., in zero electric field). Once the atoms had entered the ionization region the potential on G2 was reduced, creating a time-dependent electric field (see Fig. 2). Under these conditions the electric field experienced by the atoms does not depend on the Ps velocity distribution, and the observed time profile of the annihilation radiation is directly related to the ionization rate, which, for a given atomic state, is in turn determined by the voltage pulse ramp rate, convoluted with the detector time response. An example of annihilation radiation time profiles (cf. Fig. 4) for both static and pulsed fields is shown in Fig. 13 for $n=22$. The widths and shapes of the annihilation profiles for the three cases are very different, reflecting the different mechanisms occurring; namely, (1) atoms flying into an ionizing field at different times, (2) atoms all simultaneously experiencing a strong electric field and ionizing at the same time, and (3) atoms colliding with the vacuum chamber walls.

Since they do not depend on the Ps velocity distributions the annihilation radiation time profiles obtained using pulsed electric fields can be directly converted to yield the annihilation signal as a function of the applied electric field, as shown in Fig. 14. To better separate the time response of the annihilation signal the faster PWO detector was used to 


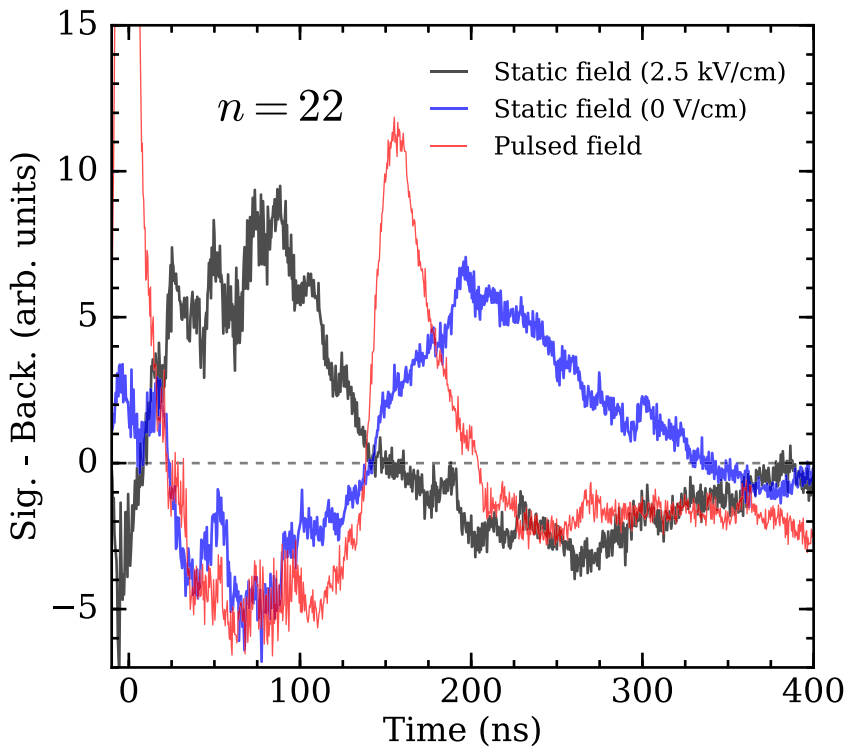

FIG. 13. Annihilation radiation time profiles for zero, static, and pulsed electric fields measured using Ps atoms with $n=22$. The pulsed electric field is turned on at approximately $t=100 \mathrm{~ns}$, as indicated in the $\mathrm{G} 2$ bias shown in Fig. 2.

perform these measurements. The time profiles were converted into electric-field profiles using the measured time dependence of the G2 voltage, an example of which is shown in Fig. 2. The HV switching time (90\%-10\%) was approximately $60 \mathrm{~ns}$, giving an electric field switching at a rate of $d F / d t \sim 35 \mathrm{~V} \mathrm{~cm}^{-1} \mathrm{~ns}^{-1}$. This rate was chosen to prevent Ps atoms from leaving the ionization region during the pulse.

The small disagreement between the simulations and measurements shown in Fig. 14 may be due to errors in the measurement of the G2 voltage time dependence. That is,

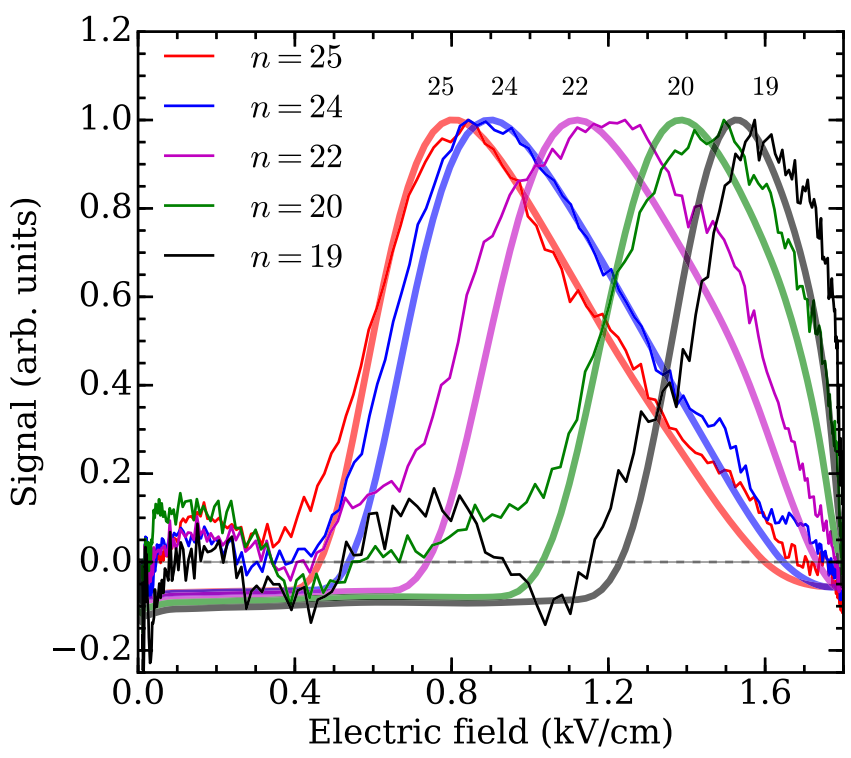

FIG. 14. Electric-field dependence of the measured pulsed-fieldionization signal (thin lines) and corresponding Monte Carlo simulations (thick lines) for atoms with different values of $n$ indicated in the legend and next to the corresponding peaks. These data were recorded by using a PWO detector. the true potential on the electrode may be shifted in time from the probe measurement. The disagreement with the calculations carried out using the methods described in Sec. III in the static field experiments is not seen in the pulsed-field measurements, since the pulsed excitation is less sensitive to small field differences, and subtleties in the ionization rates are less apparent [33] because these rates grow exponentially (see Fig. 7). This could be studied in more detail by switching the field to values where the ionization rates are slow (i.e., just below the exponential increase) and measuring the annihilation radiation signal. This method could provide a direct measurement of the annihilation rate at a well-defined field, but would require a different electrode geometry in which Ps atoms could fly unimpeded for longer. Such a modification to the apparatus would also make it possible to further distinguish between different $n$ states using pulsed electric fields by slowing down the pulse and separating the annihilation profiles, increasing the obtainable SFI state resolution.

\section{CONCLUSIONS}

We have demonstrated that the method of SFI can be applied to Ps under typical experimental conditions [34]. Measurements performed with both static and pulsed electric fields reveal clear differences in the ionization profiles for different values of $n$. Our data are generally consistent with simulations that use ionization rates obtained by scaling calculations initially developed for the hydrogen atom [35] by the Ps reduced mass. The static field measurements exhibited some discrepancies, but these are not impediments to using SFI methods in general. More precise measurements are needed to investigate the source of the disagreement. We note that recent measurements we have made of ionization rates of hydrogenic Rydberg-Stark states in helium in strong electric fields in a different apparatus also indicate discrepancies with the rates calculated using the expressions in Eqs. (5) and (6). The ionization rates measured in this work are up to five times higher than the calculated rates.

The measurements reported here are generally consistent with the theoretical calculations for higher- $n$ states, which implies that the reduced mass scaling is valid, and that there are no dynamical mechanisms that significantly change Ps tunneling rates compared with those of hydrogen. It is not necessarily obvious that Ps rates should scale from hydrogen rates directly with the reduced mass; tunneling rates depend inversely on the particle mass, and the proton and positron dynamics may be quite different during the field-ionization process. However, since the tunnel ionization rates vary exponentially with the electric field (see Fig. 7), much more precise measurements are needed to check for any difference between Ps and hydrogen; for all practical purposes the scaled hydrogen rates can be used to describe Ps experiments. It would be of interest to perform direct measurements of ionization rates using a spatially extended ionization region. In principle this would allow the application of near-threshold ionization electric fields such that the concomitant ionization rates were comparable to the Ps lifetimes [31]; for the states studied here this would correspond to rates on the order of $10-100 \mathrm{kHz}$, as opposed to $7.7 \mathrm{MHz}$. This arrangement would also be well 
suited for studies of blackbody radiation induced transitions in Rydberg Ps [17,18].

With the current SFI arrangement it would not be possible to unambiguously distinguish between neighboring $n$ states, but transitions in which $\Delta n \geqslant 2$ would be resolvable. As an example, $n=22 \rightarrow 24$ transitions, which would require $540 \mathrm{GHz}$ millimeter-wave radiation for single-photon excitation, would be observable. Electromagnetic radiation at these frequencies can be obtained from commercial sources using multipliers. Owing to the strong transition dipole moments between Rydberg levels, even two-photon transitions can be driven with typical few-mW sources that are available in the millimeter-wave range (see, e.g., Refs. [36,37]). Thus, Ps Rydberg spectroscopy using SFI in the demonstrated regime does appear to be feasible without any additional experimental modifications. Since Rydberg levels are highly sensitive to external electric and magnetic fields, microwave spectroscopy can be used to map out such external fields [38]. This would be useful for precision microwave spectroscopy of the Ps $n=2$ fine structure [39] in which Stark and Zeeman shifts must be either eliminated, or accurately taken into account [34].

Precision microwave spectroscopy of Rydberg Ps states can, in principle, be used to measure the Rydberg constant. An experiment was carried out by De Vries and Kleppner [40] in which millimeter-wave transitions between adjacent Rydberg states of atomic hydrogen were measured, and a similar measurement should be possible using Ps. If external fields are fully accounted for, the ultimate precision of such experiments would be determined by the measurement time, meaning that it would be desirable to use long-lived circular states (that is, states for which $\ell=n-1$ ) [41]. These have previously been created in hydrogen and other atoms by adiabatic transfer in crossed electric and magnetic fields [42], and by adiabatic passage from low- $\ell$ states in circularly polarized microwave fields $[41,43,44]$. Unfortunately the former technique cannot be used with Ps because the orbital magnetic moments of the oppositely charged electron and positron cancel and therefore do not given rise to a Zeeman splitting of the individual $m_{\ell}$ sublevels in the pure magnetic field. Without such a Zeeman splitting it does not appear to be possible to adiabatically transform the outer $m_{\ell}=0$ Stark state, prepared in the presence of the magnetic and strong perpendicular electric field, into the circular state in the pure magnetic field. Thus, Ps circular states will have to be generated using the microwave transfer method; SFI will be invaluable in optimizing this process [41], which will likely have to be further developed (see, e.g., Refs. [45,46]) to work within the parameters of a Ps experiment. Circular states of Ps have the advantage that, in contrast to hydrogen, they do not possess a large magnetic moment, owing to the equal and opposite orbital magnetic moments of the electron and positron. They are also naturally insensitive to electric fields because they do not exhibit static electric-dipole moments; the long lifetimes and insensitivity of circular Ps states to external magnetic and electric fields can be utilized to perform gravitational free-fall measurements $[47,48]$ and very-high-resolution time-of-flight spectroscopy [7].

The methods demonstrated here can be used to study microwave-induced transitions between Rydberg Ps levels (see, e.g., Refs. [14,49]) as part of a longer-term experimental program in which Rydberg-based field-cancellation [38] and deceleration [4] methods will enable improved optical Ps spectroscopy [50]. Precision measurements of Ps energy levels can be used to test bound-state QED theory [51], search for new physics [52], and may eventually allow for a proton-free measurement of the Rydberg constant at a level relevant to the proton radius puzzle [53,54].

\section{ACKNOWLEDGMENT}

The authors gratefully acknowledge L. Liszkay for providing silica samples. This work was supported by the EPSRC under Grant No. EP/R006474/1.
[1] D. B. Cassidy, T. H. Hisakado, H. W. K. Tom, and A. P. Mills, Jr., Optical Spectroscopy of Molecular Positronium, Phys. Rev. Lett. 108, 133402 (2012).

[2] J. Usukura and Y. Suzuki, Resonances of positronium complexes, Phys. Rev. A 66, 010502 (2002).

[3] T. F. Gallagher, Rydberg Atoms (Cambridge University Press, Cambridge, 1994).

[4] S. D. Hogan, Rydberg-Stark deceleration of atoms and molecules, EPJ Techn. Instrum. 3, 1 (2016).

[5] A. Osterwalder and F. Merkt, Using High Rydberg States as Electric Field Sensors, Phys. Rev. Lett. 82, 1831 (1999).

[6] M. Saffman, T. G. Walker, and K. Mølmer, Quantum information with Rydberg atoms, Rev. Mod. Phys. 82, 2313 (2010).

[7] A. C. L. Jones, H. J. Rutbeck-Goldman, T. H. Hisakado, A. M. Piñeiro, H. W. K. Tom, A. P. Mills, B. Barbiellini, and J. Kuriplach, Angle-Resolved Spectroscopy of Positronium Emission from a $\mathrm{Cu}(110)$ Surface, Phys. Rev. Lett. 117, 216402 (2016).

[8] A. J. McCulloch, R. W. Speirs, J. Grimmel, B. M. Sparkes, D. Comparat, and R. E. Scholten, Field ionization of Rydberg atoms for high-brightness electron and ion beams, Phys. Rev. A 95, 063845 (2017).

[9] M. P. A. Jones, L. G. Marcassa, and J. P. Shaffer, Special issue on Rydberg atom physics, J. Phys. B: At. Mol. Opt. Phys. 50, 060202 (2017).

[10] J. E. Bayfield and P. M. Koch, Multiphoton Ionization of Highly Excited Hydrogen Atoms, Phys. Rev. Lett. 33, 258 (1974).

[11] P. J. Mohr, D. B. Newell, and B. N. Taylor, Codata recommended values of the fundamental physical constants: 2014, Rev. Mod. Phys. 88, 035009 (2016).

[12] T. W. Ducas, M. G. Littman, R. R. Freeman, and D. Kleppner, Stark Ionization of High-Lying States of Sodium, Phys. Rev. Lett. 35, 366 (1975).

[13] T. F. Gallagher, L. M. Humphrey, W. E. Cooke, R. M. Hill, and S. A. Edelstein, Field ionization of highly excited states of sodium, Phys. Rev. A 16, 1098 (1977).

[14] W. E. Cooke, T. F. Gallagher, R. M. Hill, and S. A. Edelstein, Measurement of $(n d) \rightarrow(n+1) p$ intervals in sodium Rydberg states, Phys. Rev. A 16, 2473 (1977). 
[15] R. F. Stebbings, C. J. Latimer, W. P. West, F. B. Dunning, and T. B. Cook, Studies of xenon atoms in high Rydberg states, Phys. Rev. A 12, 1453 (1975).

[16] T. F. Gallagher, S. A. Edelstein, and R. M. Hill, Radiative lifetimes of the $S$ and $D$ Rydberg levels of Na, Phys. Rev. A 11, 1504 (1975).

[17] T. F. Gallagher and W. E. Cooke, Interactions of Blackbody Radiation with Atoms, Phys. Rev. Lett. 42, 835 (1979).

[18] W. E. Cooke and T. F. Gallagher, Effects of blackbody radiation on highly excited atoms, Phys. Rev. A 21, 588 (1980).

[19] T. F. Gallagher, K. A. Safinya, F. Gounand, J. F. Delpech, W. Sandner, and R. Kachru, Resonant Rydberg-atom-Rydbergatom collisions, Phys. Rev. A 25, 1905 (1982).

[20] A. Deller, B. S. Cooper, T. E. Wall, and D. B. Cassidy, Positronium emission from mesoporous silica studied by laser-enhanced time-of-flight spectroscopy, New J. Phys. 17, 043059 (2015).

[21] T. E. Wall, A. M. Alonso, B. S. Cooper, A. Deller, S. D. Hogan, and D. B. Cassidy, Selective Production of Rydberg-Stark States of Positronium, Phys. Rev. Lett. 114, 173001 (2015).

[22] R. J. Damburg and V. V. Kolosov, Theoretical studies of hydrogen Rydberg atoms in electric fields, in Rydberg States of Atoms and Molecules, edited by R. F. Stebbings and F. B. Dunning (Cambridge University Press, Cambridge, 2011).

[23] B. S. Cooper, A. M. Alonso, A. Deller, T. E. Wall, and D. B. Cassidy, A trap-based pulsed positron beam optimised for positronium laser spectroscopy, Rev. Sci. Instrum. 86, 103101 (2015).

[24] A. M. Alonso, B. S. Cooper, A. Deller, and D. B. Cassidy, Single-shot positron annihilation lifetime spectroscopy with LYSO scintillators, Nucl. Instrum. Methods Phys. Res., Sect. A 828, 163 (2016).

[25] A. P. Mills, Jr. and E. M. Gullikson, Solid neon moderator for producing slow positrons, Appl. Phys. Lett. 49, 1121 (1986).

[26] J. R. Danielson, D. H. E. Dubin, R. G. Greaves, and C. M. Surko, Plasma and trap-based techniques for science with positrons, Rev. Mod. Phys. 87, 247 (2015).

[27] D. B. Cassidy, P. Crivelli, T. H. Hisakado, L. Liszkay, V. E. Meligne, P. Perez, H. W. K. Tom, and A. P. Mills, Jr., Positronium cooling in porous silica measured via doppler spectroscopy, Phys. Rev. A 81, 012715 (2010).

[28] S. Chu and A. P. Mills, Jr., Excitation of the Positronium $1^{3} S_{1} \rightarrow 2^{3} S_{1}$ Two-Photon Transition, Phys. Rev. Lett. 48, 1333 (1982).

[29] D. B. Cassidy, S. H. M. Deng, H. K. M. Tanaka, and A. P. Mills, Jr., Single shot positron annihilation lifetime spectroscopy, Appl. Phys. Lett. 88, 194105 (2006).

[30] D. B. Cassidy and A. P. Mills, Jr., A fast detector for singleshot positron annihilation lifetime spectroscopy, Nucl. Instrum. Methods Phys. Res., Sect. A 580, 1338 (2007).

[31] A. Deller, A. M. Alonso, B. S. Cooper, S. D. Hogan, and D. B. Cassidy, Measurement of Rydberg positronium fluorescence lifetimes, Phys. Rev. A 93, 062513 (2016).

[32] A. M. Alonso, B. S. Cooper, A. Deller, S. D. Hogan, and D. B. Cassidy, Positronium decay from $n=2$ states in electric and magnetic fields, Phys. Rev. A 93, 012506 (2016).

[33] W. E. Cooke and T. F. Gallagher, Dependence of Rydbergstate field-ionization thresholds on $\left|m_{\ell}\right|$, Phys. Rev. A 17, 1226 (1978).
[34] D. B. Cassidy, Experimental progress in positronium laser physics, Eur. Phys. J. D 72, 53 (2018).

[35] R. J. Damburg and V. V. Kolosov, A hydrogen atom in a uniform electric field. III, J. Phys. B: At. Mol. Phys. 12, 2637 (1979).

[36] E. S. Shuman, J. Nunkaew, and T. F. Gallagher, Two-photon microwave spectroscopy of ba $6 s n l$ states, Phys. Rev. A 75, 044501 (2007).

[37] D. A. Tate and T. F. Gallagher, Microwave-optical twophoton excitation of Rydberg states, Phys. Rev. A 97, 033410 (2018).

[38] A. Deller and S. D. Hogan, Microwave spectroscopy of the $1 s n p^{3} P_{J}$ fine structure of high Rydberg states in ${ }^{4} \mathrm{He}$, Phys. Rev. A 97, 012505 (2018).

[39] D. Hagena, R. Ley, D. Weil, G. Werth, W. Arnold, and H. Schneider, Precise Measurement of $n=2$ Positronium FineStructure Intervals, Phys. Rev. Lett. 71, 2887 (1993).

[40] J. C. De Vries, A precision millimeter-wave measurement of the Rydberg frequency, Ph.D. thesis, Massachusetts Institute of Technology, 2001.

[41] R. G. Hulet and D. Kleppner, Rydberg Atoms in "Circular" States, Phys. Rev. Lett. 51, 1430 (1983).

[42] D. Delande and J. C. Gay, A new method for producing circular Rydberg states, Europhys. Lett. 5, 303 (1988).

[43] C. H. Cheng, C. Y. Lee, and T. F. Gallagher, Production of Circular Rydberg States with Circularly Polarized Microwave Fields, Phys. Rev. Lett. 73, 3078 (1994).

[44] R. Lutwak, J. Holley, P. P. Chang, S. Paine, D. Kleppner, and T. Ducas, Circular states of atomic hydrogen, Phys. Rev. A 56, 1443 (1997).

[45] A. Signoles, E. K. Dietsche, A. Facon, D. Grosso, S. Haroche, J. M. Raimond, M. Brune, and S. Gleyzes, Coherent Transfer between Low-Angular-Momentum and Circular Rydberg States, Phys. Rev. Lett. 118, 253603 (2017).

[46] S. Patsch, D. M. Reich, J.-M. Raimond, M. Brune, S. Gleyzes, and C. P. Koch, Fast and accurate circularization of a Rydberg atom, Phys. Rev. A 97, 053418 (2018).

[47] A. P. Mills, Jr. and M. Leventhal, Can we measure the gravitational free fall of cold Rydberg state positronium? Nucl. Instrum. Methods Phys. Res., Sect. B 192, 102 (2002).

[48] D. B. Cassidy and S. D. Hogan, Atom control and gravity measurements using Rydberg positronium, Int. J. Mod. Phys.: Conf. Ser. 30, 1460259 (2014).

[49] K. A. Safinya, T. F. Gallagher, and W. Sandner, Resonance measurements of $f-h$ and $f-i$ intervals in cesium using selective and delayed field ionization, Phys. Rev. A 22, 2672 (1980).

[50] M. S. Fee, S. Chu, A. P. Mills, R. J. Chichester, D. M. Zuckerman, E. D. Shaw, and K. Danzmann, Measurement of the positronium $1^{3} S_{1}-2^{3} S_{1}$ interval by continuous-wave two-photon excitation, Phys. Rev. A 48, 192 (1993).

[51] S. G. Karshenboim, Precision study of positronium: Testing bound state QED theory, Int. J. Mod. Phys. A 19, 3879 (2004).

[52] M. S. Safronova, D. Budker, D. DeMille, D. F. Jackson Kimball, A. Derevianko, and C. W. Clark, Search for new physics with atoms and molecules, Rev. Mod. Phys. 90, 025008 (2018). 
[53] A. Beyer, L. Maisenbacher, A. Matveev, R. Pohl, K. Khabarova, A. Grinin, T. Lamour, D. C. Yost, T. W. Hänsch, N. Kolachevsky, and T. Udem, The Rydberg constant and proton size from atomic hydrogen, Science 358, 79 (2017).
[54] H. Fleurbaey, S. Galtier, S. Thomas, M. Bonnaud, L. Julien, F. Biraben, F. Nez, M. Abgrall, and J. Guéna, New Measurement of the $1 s-3 s$ Transition Frequency of Hydrogen: Contribution to the Proton Charge Radius Puzzle, Phys. Rev. Lett. 120, 183001 (2018). 